\title{
Comparison between Fourth and Second Order DT-MR image Segmentations
}

\author{
Saba El-Hilo ${ }^{1}$, Yonas T. Weldeselassie ${ }^{1}$, and M. Stella Atkins ${ }^{1}$ \\ ${ }^{1}$ Medical Image Analysis Lab, School of Computing Science Simon Fraser University, Burnaby, \\ BC V5A 1S6, Canada;
}

\begin{abstract}
A second order tensor is usually used to describe the diffusion of water for each voxel within a Diffusion Tensor Magnetic Resonance (DT-MR) images. However, a second order tensor approximation fails to accurately represent complex local tissue structures such as crossing fibers. Therefore, higher order tensors are used to represent more complex diffusivity profiles. In this work we examine and compare segmentations of both second order and fourth order DT-MR images using the Random Walker segmentation algorithm with the emphasis of pointing the shortcomings of second order tensor model in segmenting regions with complex fiber structures. We first adopt the Random Walker algorithm for segmenting diffusion tensor data by using appropriate tensor distance metrics and then demonstrate the advantages of performing segmentation on higher order DT-MR data. The approach proposed takes advantage of all the information provided by the tensors by using suitable tensor distance metrics. The distance metrics used are: the Log-Euclidean for the second order tensors and the normalized $L_{2}$ distance for the fourth order tensors. The segmentation is carried out on a weighted graph that represents the image where, the tensors are the nodes and the edge weights are computed using the tensor distance metrics. Applying the approach to both synthetic and real DT-MRI data yields segmentations that are both robust and qualitatively accurate.
\end{abstract}

Keywords: Segmentation, diffusion tensor magnetic resonance imaging, random walker, tensor distance metric, second order, fourth order

\section{INTRODUCTION}

Diffusion tensor magnetic resonance imaging (DT-MRI) has attracted attention in the field of medical imaging as it remains the only non invasive method that can reveal the structure of white matter anatomy. It also provides information about tissue orientation and microstructural integrity.

The DT-MR imaging technique is based on the anisotropy of water self diffusion. The anisotropic nature of diffusion of water molecules in tissues has been described mathematically by a rank- 2 tensor where at each voxel of the imaging volume is a $2^{\text {nd }}$ order $3 \times 3$ symmetric positive definite matrix (diffusion tensor) which depicts the anisotropy within the tissue. Scalar measures such as fractional anisotropy computed from $2^{\text {nd }}$ order tensors have been commonly used to approximate the diffusivity of water in tissue in vivo. ${ }^{1}$ However, the $2^{\text {nd }}$ order tensor model fails to correctly represent regions with complex local tissue structures such as crossing and merging of fiber bundles. The model fails to correctly represent the anisotropy in areas of fiber crossing which is evident by the significant drop in the value of fractional anisotropy in these areas.

Several approaches have been proposed to overcome the limitation of $2^{\text {nd }}$ order tensors. Tuch et al. ${ }^{2}$ proposed a method known as high angular resolution diffusion imaging (HARDI). The method performs diffusion imaging with diffusion weighted gradients along many directions that are distributed isotropically on the surface of a unit sphere. Multiple fiber components are identified by calculating the probability distribution function (PDF) of the diffusion process in each voxel. However, this method is time intensive and therefore, is impractical for clinical use.

Another approach that tends to be more practical is describing the apparent diffusion coefficient (ADC) by higher order tensors (e.g. $\left.4^{\text {th }}\right){ }^{3,4}$ Higher order tensors generalize the second-order tensors and have the ability to

S.E, Y.T.W, M.S.A: E-mail: selhilo, yonas, stella@sfu.ca 
represent more complex tissue geometry with multi-lobe diffusivity profiles. This approach is especially attractive because the algorithms and metrics developed for second order tensors can be extended to higher order tensors.

DT-MRI image analysis features several open research problems in the areas of visualization, regularization, restoration and segmentation. Image segmentation is the process of separating an image into regions of interest. Several methods have been proposed that address DT-MR image segmentation. The method proposed by Zhukov et al. ${ }^{5}$ focuses on segmenting white matter regions in DT-MR images. The method uses a scalar measure and in return, ignores the directional information contained in the tensors. Therefore, the algorithm fails to distinguish between regions which have the same diffusion anisotropy magnitude but different directions. The method presented by Wang and Vemuri ${ }^{6}$ uses both directions and magnitude of diffusion to define a region based force. However, the method is sensitive to parameter initialization and has the risk of finding suboptimal solutions. Weldeselassie and Hamarneh ${ }^{7}$ also use the whole tensor information to perform segmentation using graph-cuts approach. However, the graph cuts segmentation method suffers from the small cut problem and doesn't extend easily to more than two labels.

In this work, we extend the random walker segmentation algorithm ${ }^{8}$ proposed for the segmentation of scalar images to segment both second and fourth order DT-MR image data. The random walker segmentation technique provides a k-way image segmentation given user labeled voxels called seeds. The seeds are used to define a region of interest which needs to be segmented. A segmentation is produced on a weighted graph by computing the probability that a random walker starting its walk at a voxel first reaches a seed with a label where, each voxel in the image is assigned the label with the greatest probability. The DT-MRI data is converted to a graph where each tensor is a vertex and is connected to neighboring tensors by a weighted edge. Tensor dissimilarity metrics are used to asses the similarity between tensors and calculate the weights on the edges of the graph. The metric used for $2^{\text {nd }}$ order tensors is the Log-Euclidean ${ }^{9}$ whereas the $L_{2}$ distance $^{3}$ is used for the $4^{\text {th }}$ order tensors. Moreover, we demonstrate the advantages of performing segmentation on higher order data.

The paper is organized as follows. In section 2.1 we provide an overview of the random walker formulation and its extension to segment DT-MRI data. In section 2.2 we present the tensor dissimilarity metrics used. Section 2.3 describes how the weights are calculated. We present experimental segmentation results on both synthetic and real DT-MRI data in section 3. We summarize and draw concluding remarks in section 4.

\section{METHODS}

\subsection{Random walker formulation}

The segmentation is carried out on a weighted graph which is constructed from the image to be segmented. A graph consists of a pair $\mathrm{G}=(\mathrm{V}, \mathrm{E})$ with vertices $v \in V$ and edges $e \in E$. An edge between two vertices $v_{i}$ and $v_{j}$ is referred to as $e_{i j}$ whereas, the weight of the edge is referred to as $w_{i j}$. Each vertex in the graph is a tensor voxel $\left(T_{i}\right)$ from the DT-MR image. Neighboring tensors $\left(T_{i}, T_{j}\right)$ are connected by edges $e_{i j}$ which have weights $w_{i j}$. The weights of the edges are computed using the tensor distance measures. The Log Euclidean is used for the second order datasets whereas the $L_{2}$ distance is used for the fourth order datasets. An edge connecting two similar tensors should have a larger weight than an edge connecting to dissimilar tensors.

The user provides a set of labeled voxels $V_{L}$ with $\mathrm{K}$ labels which are referred to as seeds. For instance, the user can provide two types of labels $(\mathrm{K}=2)$ : object and background, where the object label constitutes of the tensors that belong to the object to be segmented and the background label corresponds to the tensors that make up the background of the DT-MR image. Therefore, given the graph and a set of labeled vertices, the goal of the random walker algorithm is to label each unlabeled vertex $V_{U}$ in the graph with a label $s$ provided by the user. $^{8}$

For each unlabeled vertex $v_{i} \in V_{U}$ a probability $x_{i}^{s}$ is calculated which represents the probability that a random walker starting from that vertex first reaches a labeled vertex $v_{j} \in V_{L}$ assigned to label $s$. The segmentation is then produced by labeling each vertex with the label for which it has the highest probability. To find the probability $x_{i}^{s}$ that a random walker starting from a vertex $v_{i}$ first reaches a labeled node, the following energy functional has to be minimized ${ }^{8}$

$$
E=x_{U}^{s T} L x_{U}^{s}
$$


where $s$ corresponds to a label and L represents the combinatorial Laplacian matrix defined as

$$
L_{v_{i}, v_{j}}=\left\{\begin{aligned}
\operatorname{deg}_{v_{i}} & \text { if } i=j \\
-w_{i j} & \text { if } v_{i} \text { and } v_{j} \text { are adjacent vertices } \\
0 & \text { otherwise }
\end{aligned}\right.
$$

where $\operatorname{deg}_{v_{i}}$ is the degree of the vertex $v_{i}$. The Laplacian matrix can be partitioned into labeled (L) and unlabeled (U) vertices

$$
L=\left[\begin{array}{cc}
L_{L} & B \\
B^{T} & L_{U}
\end{array}\right]
$$

The minimization of the energy function in Eq. 1 can then be achieved by solving the following system of equations

$$
L_{U} x_{U}^{s}=-B f^{s}
$$

where $f^{s}$ is an indicator vector for the labeled vertices that defines the segmentation boundary.

\subsection{Tensor distance metrics}

The edge weights of the constructed graph represent how similar or dissimilar two vertices are. When dealing with diffusion tensors appropriate tensor distance metrics have to be used. More specifically, the metrics should utilize all the information provided by the tensors. So, it should incorporate both the diffusion magnitude (eigenvalues) and direction (eigenvectors).

The Log-Euclidean tensor distance $\left(d_{L E}\right)^{9}$ performs Euclidean calculations in the domain of matrix logarithms and can be used to find the distance between two second order tensors $\left(T_{i}, T_{j}\right)$

$$
d_{L E}\left(T_{i}, T_{j}\right)=\sqrt{\operatorname{tr}\left(\left(\log \left(T_{i}\right)-\log \left(T_{j}\right)\right)^{2}\right)}
$$

where $\operatorname{tr}($.$) is the trace of the matrix. On the other hand, a distance measure between 4^{\text {th }}$ order diffusion tensors $\left(D_{i}, D_{j}\right)$ can be computed using the normalized $L_{2}$ norm ${ }^{3}$ between the corresponding diffusivity functions $d_{1}(g)$ and $d_{2}(g)$. The diffusivity function is first represented using a $4^{\text {th }}$ order tensor as:

$$
d(g)=\sum_{i+j+k=4} D_{i, j, k} g_{1}^{i} g_{2}^{j} g_{3}^{k}
$$

where $g=\left[g_{1}, g_{2}, g_{3}\right]^{T}$ is the magnetic field gradient direction. Diffusion is symmetric therefore, the $4^{\text {th }}$ order model results in a $3 \times 3 \times 3 \times 3$ totally symmetric positive definite matrix with 15 unique coefficients; whereas the $2^{\text {nd }}$ order model results in a $3 \times 3$ symmetric positive definite matrix with 6 unique coefficients.

The normalized $L_{2}$ distance between two $4^{\text {th }}$ order diffusivity functions is computed using the following equation $^{3}$

$$
\begin{aligned}
d_{L 2}^{2}\left(D_{1}, D_{2}\right)= & \frac{1}{4 \pi} \int_{s^{2}}\left[d_{1}(g)-d_{2}(g)\right]^{2} d g \\
& =\frac{1}{315}\left[\left(\Delta_{4,0,0}+\Delta_{0,4,0}+\Delta_{0,0,4}+\Delta_{2,2,0}+\Delta_{0,2,2}+\Delta_{2,0,2}\right)^{2}+4\left[\left(\Delta_{4,0,0}+\Delta_{2,2,0}\right)^{2}\right.\right. \\
& +\left(\Delta_{4,0,0}+\Delta_{2,0,2}\right)^{2}+\left(\Delta_{4,0,0}+\Delta_{2,2,0}\right)^{2}+\left(\Delta_{0,4,0}+\Delta_{0,2,2}\right)^{2}+\left(\Delta_{0,0,4}+\Delta_{0,2,2}\right)^{2} \\
& \left.+\left(\Delta_{0,0,4}+\Delta_{2,0,2}\right)^{2}\right]+24\left(\Delta_{4,0,0}^{2}+\Delta_{0,4,0}^{2}+\Delta_{0,0,4}^{2}\right)-6\left(\Delta_{2,2,0}^{2}+\Delta_{0,2,2}^{2}+\Delta_{2,0,2}^{2}\right) \\
& +2\left(\Delta_{4,0,0}+\Delta_{0,4,0}+\Delta_{0,0,4}\right)^{2}+\left(\Delta_{2,1,1}+\Delta_{0,3,1}+\Delta_{0,1,3}\right)^{2}+\left(\Delta_{1,2,1}+\Delta_{3,0,1}+\Delta_{1,0,3}\right)^{2} \\
& +\left(\Delta_{1,1,2}+\Delta_{3,1,0}+\Delta_{1,3,0}\right)^{2}+2\left[\left(\Delta_{3,1,0}+\Delta_{1,3,0}\right)^{2}+\left(\Delta_{3,0,1}+\Delta_{1,0,3}\right)^{2}+\left(\Delta_{0,3,1}+\Delta_{0,1,3}\right)^{2}\right] \\
& \left.+2\left(\Delta_{3,1,0}^{2}+\Delta_{3,0,1}^{2}+\Delta_{1,3,0}^{2}+\Delta_{0,3,1}^{2}+\Delta_{1,0,3}^{2}+\Delta_{0,1,3}^{2}\right)\right]
\end{aligned}
$$


where the integral is over all unit vectors and the coefficients $\Delta_{i, j, k}$ are computed by subtracting the coefficients $D_{i, j, k}$ of the tensor $D_{i}$ from the corresponding coefficients of the tensor $D_{j}$. In this work, we denote the coefficients of the $4^{t h}$ order diffusion tensor using the notation used in, ${ }^{3}$ i.e., $D_{i, j, k}$, where $i+j+k=4$. The integral in equation 6 can be computed analytically and the result can be expressed as a sum of squares of the terms $\Delta_{i, j, k}$ as shown in Eq. 8. This makes the implementation of the distance measure between $4^{\text {th }}$ order tensors efficient. Moreover, the distance measure is invariant to rotations since the integral is defined over all gradient directions.

\subsection{Calculating the weights}

Using the distance metrics the edge weights on the graph can be calculated using the following equation,

$$
w_{i j}=e^{-\beta d\left(T_{i}, T_{j}\right)^{2}}+\epsilon
$$

where $\epsilon$ is a small constant and $\beta$ is a free scaling parameter set by the user. $d$ is $d_{L E}\left(T_{i}, T_{j}\right)$ when performing segmentation on second order tensors and $d_{L 2}\left(D_{i}, D_{j}\right)$ when performing segmentation on fourth order tensors. The tensor distances are normalized to a range of $[0,1]$.

\section{RESULTS}

Segmentations were performed on both second and fourth order DT-MR images using the above methodology. Positive definite tensors of second and fourth order were estimated using the method proposed by Barmpoutis et al. ${ }^{4}$ Segmentation results of both second and fourth order datasets are presented for comparison purposes. The advantage of performing segmentation on fourth order datasets instead of second order is demonstrated.

Standard hard constraints were used in all the examples. Both background and object seeds were provided as an input. For all the segmentation results, the object seeds are shown in green and the background seeds in yellow. Moreover, the DT-MR images sets are visualized using the trace. For our experiments the $\beta$ parameter in Eq. 9 had a noticeable effect on the results of the segmentations. The optimal $\beta$ parameter in our experiments was determined by trial and error. Multiple values were tested until a qualitatively satisfactory segmentation was achieved.

\subsection{Synthetic data sets}

Our synthetic dataset presented here is of size 100x100 and contains a 40x40 box which is composed of simulated crossing fibers with orientations of $[0.70710 .70710]$ and $\left[\begin{array}{lllll}0.7071 & -0.7071 & 0\end{array}\right]$. The box is surrounded by simulated crossing fibers with orientations of $\left[\begin{array}{lll}1 & 0 & 0\end{array}\right]$ and $\left[\begin{array}{lll}0 & 1 & 0\end{array}\right]$. Fig. 1 shows both the second order and fourth order diffusion tensor fields reconstructed from the same synthetic image slice and are visualized via a spherical visualization technique ${ }^{*}$. The second order approximation fails to correctly represent the fiber crossings and instead shows all the tensors in the image as being planar. It fails to distinguish the inner box from the surrounding fibers. Whereas, the fourth order approximation can differentiate between both regions and correctly displays the crossing fibers. Note that to avoid tensor cluttering, we plot the tensors at intervals of 10.

Fig. 2 shows the segmentation result of the synthetic dataset, where the aim is to segment the middle black box from the white background. The segmented result is shown by red outline. Clearly, the second order model fails to segment the middle box because as shown in fig. 2(b) the second order tensor model lacks the ability to distinguish between crossing fibers. Whereas the fourth order model accurately segments the middle box as shown in fig. 2(c). This also serves to demonstrate that the normalized $L_{2}$ distance metric takes into account both the direction and the magnitude of the $4^{\text {th }}$ order diffusion tensors.

\subsection{Real data sets}

The proposed segmentation technique was also tested on a real medical data set. The dataset presented here consists of a human brain where 99 Diffusion Weighted (DW) volumes are acquired in 99 gradient directions. There is one $S_{0}$ volume acquired with no gradient direction and the B-value is 3000 .

\footnotetext{
${ }^{*}$ The visualization is performed using a computer program implemented by Barmpoutis and is available at: http://www.cise.ufl.edu/ abarmpou/lab/
} 


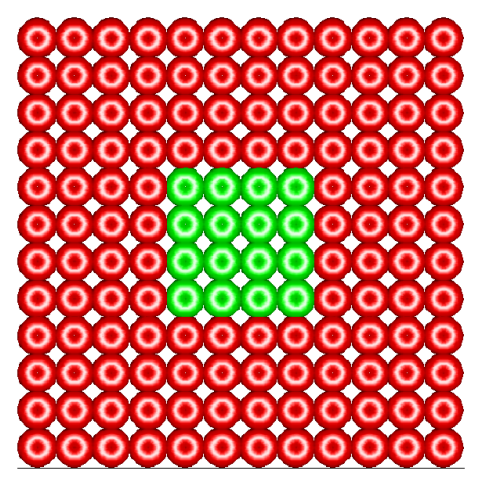

(a) $2^{\text {nd }}$ order tensors

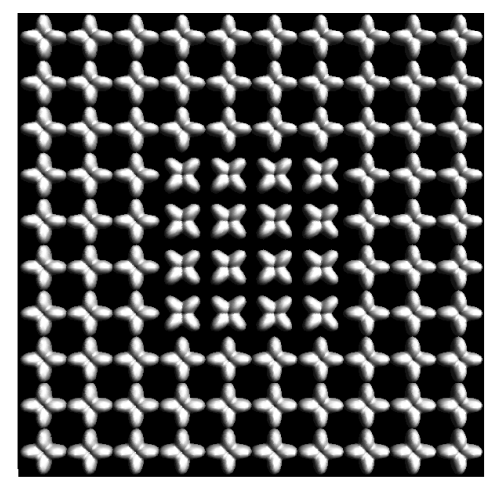

(b) $4^{\text {th }}$ order tensors

Figure 1. Spherical visualization of the synthetic data set.

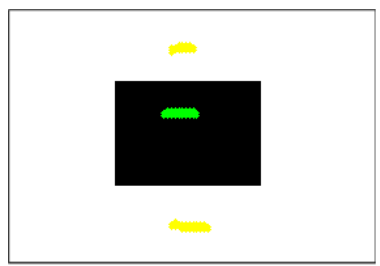

(a) Initial image with seeds

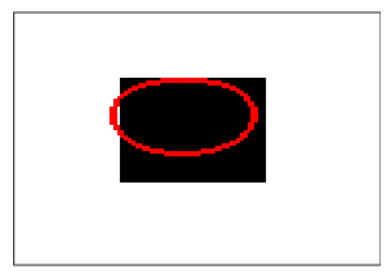

(b) $2^{\text {nd }}$ order tensors segmentation

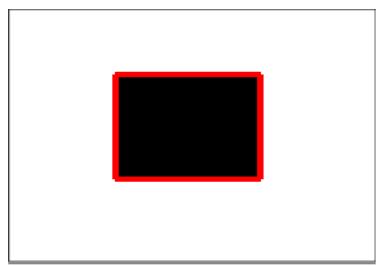

(c) $4^{\text {th }}$ order tensors segmentation

Figure 2. Segmentation result of the synthetic data set.

\subsubsection{Regions of interest to segment}

Anatomic areas where the local fiber distribution is characterized as an intersection of fibers are chosen to be segmented. These regions are selected to demonstrate the advantage of performing segmentations on $4^{\text {th }}$ order datasets. The following areas were chosen that show fiber crossing: the transverse view of the crossing between the corona radiata and corpus callosum, the coronal view of the same crossing and the lateral view of the corpus callosum. ${ }^{10}$ The segmentation technique is applied to all these three scenerios where the corpus callosum is the object of the segmentation.

Fig. 3 displays the segmentation result of the first region which is the transverse view of the crossing between the corona radiata and corpus callosum. Only the left part of the corpus callosum was marked with seeds. The $2^{\text {nd }}$ order model (fig. 3(b)) segmented the left side correctly but failed to segment the right side which could be attributed to the presence of crossing fibers in that area. The inaccuracy of the segmentation result can be overcome by placing more seeds on the corpus callosum area. The $4^{\text {th }}$ order model (fig. 3(c)) accurately segmented both sides of the corpus callosum even with only one side seeded.

Fig. 4 displays the segmentation result of the second region which is the coronal view of the crossing between the corona radiata and the corpus callosum. Again, only one side of the corpus callosum is marked with seeds. The $2^{\text {nd }}$ order model (fig. 4(b)) failed to segment the corpus callosum accurately. Whereas, the $4^{\text {th }}$ order model (fig. 4(c)) segmented the corpus callosum area accurately.

Fig. 5 displays the segmentation result of the corpus callosum from the lateral view. More seeds were initialized than the two examples above(fig. 3 and fig. 4) to demonstrate that the $2^{\text {nd }}$ order model can produce an accurate segmentation when additional seeds are placed. The $2^{\text {nd }}$ order model produced a segmentation result similar to the $4^{\text {th }}$ order model. However, the $4^{\text {th }}$ order model still produced a qualitatively more accurate segmentation. 


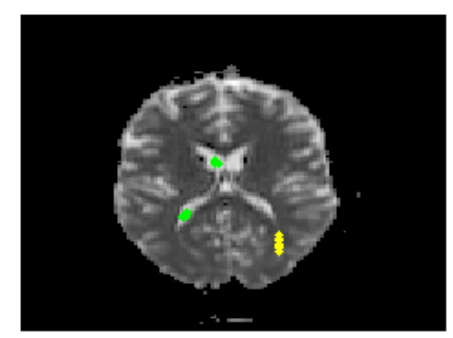

(a) Initial image with seeds

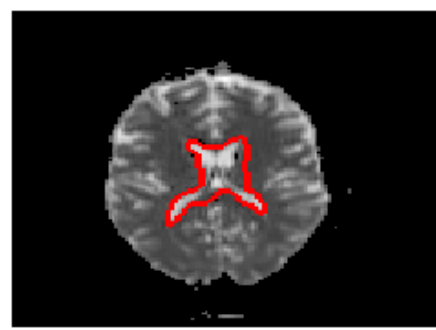

(b) $2^{\text {nd }}$ order tensors segmentation

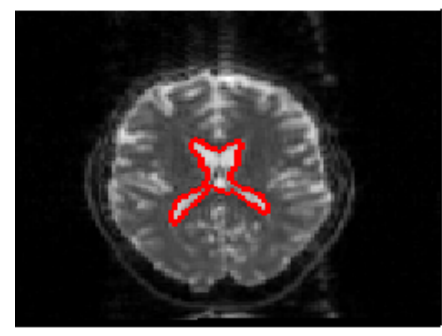

(c) $4^{\text {th }}$ order tensors segmentation

Figure 3. Segmentation result of the corpus callosum from the transverse view.

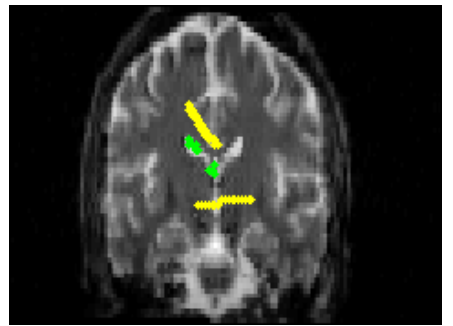

(a) Initial image with seeds

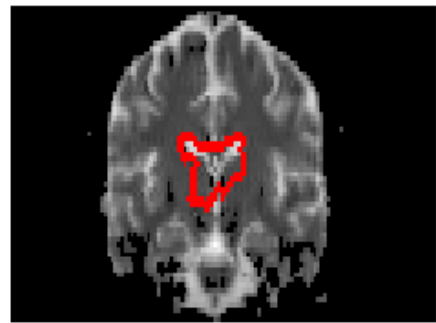

(b) $2^{\text {nd }}$ order tensors segmentation

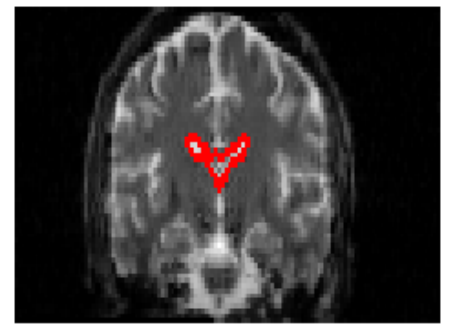

(c) $4^{\text {th }}$ order tensors segmentation

Figure 4. Segmentation result of the corpus callosum from the coronal view .

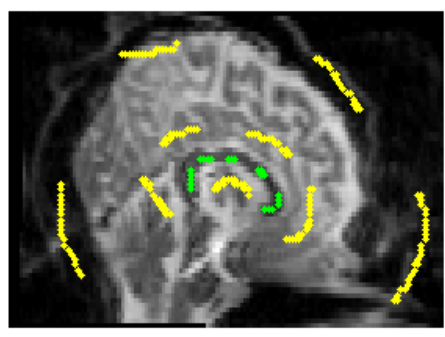

(a) Initial image with seeds

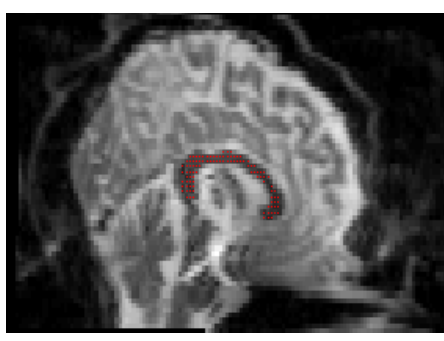

(b) $2^{\text {nd }}$ order tensors segmentation

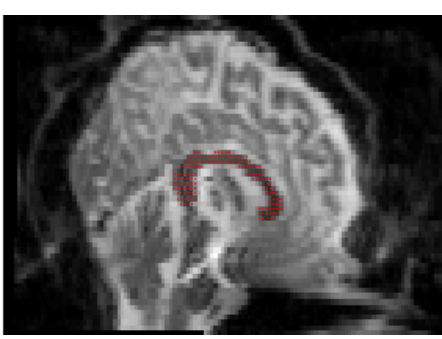

(c) $4^{\text {th }}$ order tensors segmentation

Figure 5. Segmentation result of the corpus callosum from the lateral view. Demonstration of segmentation result when more seeds are initialized 


\section{CONCLUSIONS}

The random walker segmentation method has been applied to scalar images and has lead to robust results. We have shown in this work that the random walker segmentation algorithm can be extended to segment both $2^{\text {nd }}$ and $4^{t h}$ order DT-MR images by utilizing appropriate tensor distance metrics. The segmentation is carried out on a weighted graph where, tensor distance metrics that use the full tensor information are used to define edge weights. The normalized $L_{2}$ tensor distance is used to define the weights on the graph for the $4^{\text {th }}$ order tensor field segmentation whereas the Log-Euclidean is used for the $2^{\text {nd }}$ order tensor field segmentation.

The proposed approach is applied on both real and synthetic DT-MR images. The synthetic data set was constructed to demonstrate the limitation of the $2^{\text {nd }}$-order tensors to correctly represent fiber crossings. In all the experiments carried out, the $2^{\text {nd }}$ order tensor model lead to inaccurate and poor segmentation results in regions with complex fiber structures as compared to the segmentation results obtained from the $4^{\text {th }}$ order tensor data. This demonstrates the advantage of performing segmentation on higher order tensors.

Our segmentation results were very sensitive to the free $\beta$ parameter that is used in Eq. 9 and also to the number of seeds initially placed by the user. This is especially the case for the $2^{\text {nd }}$ order tensor field segmentation where the placement of more seeds resulted in more accurate segmentations. This is a limitation of the underlying random walker segmentation technique. Future work will investigate how to automatically select a suitable value for $\beta$ and test the segmentation technique on more real datasets in addition to quantitatively compare the segmentation results obtained from $2^{\text {nd }}$ order and $4^{\text {th }}$ order tensor fields.

\section{ACKNOWLEDGMENTS}

We would like to thank Leo Grady for making the random walker segmentation computer program for segmenting scalar images available for the public which we used in our work ${ }^{\dagger}$. Our thanks also goes to Dr. Angelos Barmpoutis for making the FanDTasia tool available which was used to estimate $4^{\text {th }}$ order tensor fields and for the processing of our DT-MRI data ${ }^{\ddagger}$. This work was supported by the Natural Sciences and Engineering Research Council of Canada (NSERC).

\section{REFERENCES}

[1] Westin, C., Maier, S., Mamata, H., Nabavi, A., Jolesz, F., and Kikinis, R., "Processing and visualization for diffusion tensor MRI," Medical Image Analysis 6(2), 93-108 (2002).

[2] Tuch, D., Reese, T., Wiegell, M., Makris, N., Belliveau, J., and Wedeen, V., "High angular resolution diffusion imaging reveals intravoxel white matter fiber heterogeneity," Magnetic Resonance in Medicine 48(4), 577-582 (2002).

[3] Barmpoutis, A., Hwang, M., Howland, D., Forder, J., and Vemuri, B., "Regularized positive-definite fourth order tensor field estimation from DW-MRI," NeuroImage 45(1), S153-S162 (2009).

[4] Barmpoutis, A. and Vemuri, B., "A unified framework for estimating diffusion tensors of any order with symmetric positive-definite constraints," in [Biomedical Imaging: From Nano to Macro, 2010 IEEE International Symposium on], 1385-1388, IEEE (2010).

[5] Zhukov, L., Museth, K., Breen, D., Whitaker, R., and Barr, A., "Level set modeling and segmentation of DT-MRI brain data," Journal of Electronic Imaging 12(1), 125-133 (2003).

[6] Wang, Z. and Vemuri, B., "An affine invariant tensor dissimilarity measure and its applications to tensorvalued image segmentation," in [Computer Vision and Pattern Recognition, 2004. CVPR 2004. Proceedings of the 2004 IEEE Computer Society Conference on], 1, IEEE (2004).

[7] Weldeselassie, Y. and Hamarneh, G., "DT-MRI segmentation using graph cuts," in [Proc. of SPIE Vol], 6512, $65121 \mathrm{~K}-1$, Citeseer.

[8] Grady, L., "Random walks for image segmentation," IEEE Transactions on Pattern Analysis and Machine Intelligence, 1768-1783 (2006).

\footnotetext{
${ }^{\dagger}$ available at: http://cns.bu.edu/lgrady/software.html

${ }^{\ddagger}$ Available at: http://www.cise.ufl.edu/ abarmpou/lab/
} 
[9] Arsigny, V., Fillard, P., Pennec, X., and Ayache, N., "Fast and simple calculus on tensors in the LogEuclidean framework," Medical Image Computing and Computer-Assisted Intervention-MICCAI 2005 , $115-122(2005)$.

[10] Wiegell, M., Larsson, H., and Wedeen, V., "Fiber crossing in human brain depicted with diffustion tensor MR imaging," Radiology 217, 897-903 (2000). 